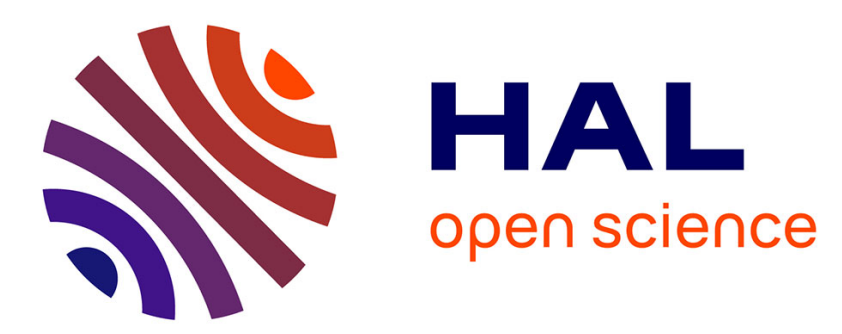

\title{
Band structure of magnetic excitations in the vortex phase of a ferromagnetic superconductor
}

\author{
A. A. Bespalov, Alexandre I. Buzdin
}

\section{To cite this version:}

A. A. Bespalov, Alexandre I. Buzdin. Band structure of magnetic excitations in the vortex phase of a ferromagnetic superconductor. Physical Review B: Condensed Matter and Materials Physics (1998-2015), 2013, 87 (9), pp.094509. 10.1103/PhysRevB.87.094509 . hal-00814226

\section{HAL Id: hal-00814226 \\ https://hal.science/hal-00814226}

Submitted on 28 Feb 2018

HAL is a multi-disciplinary open access archive for the deposit and dissemination of scientific research documents, whether they are published or not. The documents may come from teaching and research institutions in France or abroad, or from public or private research centers.
L'archive ouverte pluridisciplinaire HAL, est destinée au dépôt et à la diffusion de documents scientifiques de niveau recherche, publiés ou non, émanant des établissements d'enseignement et de recherche français ou étrangers, des laboratoires publics ou privés. 


\title{
Band structure of magnetic excitations in the vortex phase of a ferromagnetic superconductor
}

\author{
A. A. Bespalov ${ }^{1,2}$ and A. I. Buzdin ${ }^{2}$ \\ ${ }^{1}$ Institute for Physics of Microstructures, Russian Academy of Sciences, GSP-105, 603950, Nizhny Novgorod, Russia \\ ${ }^{2}$ Université Bordeaux, LOMA, UMR 5798, F-33600 Talence, France
}

(Received 16 January 2013; published 18 March 2013)

\begin{abstract}
Magnetic excitations in a ferromagnetic superconductor in the presence of an Abrikosov vortex lattice have been studied using the phenomenological London and Landau-Lifshitz equations. Due to the periodicity of the vortex field the magnon spectrum has a band structure, similar to the structure of the electon spectrum in a crystal lattice. The gaps between adjacent bands have been calculated using an analog of the weak-binding approximation. When the applied magnetic field is altered the band structure undergoes a qualitative transformation due to commensurability effects, connected with the nonmonotonicity of the magnon spectrum in the Meissner state. In dirty samples the energy gaps may be smeared out because of the dissipation connected with vortex motion. In sufficiently clean samples the gaps manifest themselves as maxima in the frequency dependence of the microwave reflectivity coefficient.
\end{abstract}

DOI: 10.1103/PhysRevB.87.094509

PACS number(s): 75.30.Ds, 74.25.Uv, 74.20.De

\section{INTRODUCTION}

The discovery of ferromagnetic superconductors ${ }^{1-5}$ provided a unique opportunity to study the interplay between magnetism and triplet superconductivity in one compound. However, the investigation of magnetic properties in the superconducting state of these materials is hampered by the Meissner effect, consisting in the screening of static magnetic fields. Still, dynamical measurements can be applied, for example, microwave probing of the spin wave spectrum. ${ }^{6}$ Spin waves in magnetic superconductors in the Meissner state have been studied theoretically, using different phenomenological approaches, in several papers. ${ }^{6-10}$ Buzdin $^{7}$ determined the magnon spectrum in an antiferromagnetic superconductor with an easy-axis anisotropy. Braude and Sonin ${ }^{6,9}$ calculated the magnon spectrum and the microwave response of a ferromagnetic supercondutor. In Refs. 8 and 10 two-dimensional magnons on domain walls and on the surface of the ferromagnet have been studied.

Experimental measurements of the ac magnetic susceptibility of superconducting ferromagnets revealed that the ideal diamagnetic response is not reached in the superconducting phase. ${ }^{2-4}$ This observation allows to suggest that these materials are always found in the spontaneous mixed state due to the presence of the intrinsic field created by the magnetization. Thus, the theoretical study of magnetic excitations in the vortex phase is also relevant. The influence of Abrikosov vortices on magnetization dynamics in magnetic superconductors has been considered in a number of theoretical papers. In Refs. 11-13 it has been demonstrated that the magnon spectrum can be examined using the vortex motion induced by a dc or ac transport current. The contribution to the vortex viscosity connected with dissipation due to the Cherenkov radiation of magnons has been determined. In Ref. 14 it has been predicted that the flux flow should lead to the creation of domain walls in systems with slow relaxation of the magnetic moments. $\mathrm{Ng}$ and Varma ${ }^{15}$ considered coupled spin and vortex dynamics in ferromagnetic superconductors in the absence of a transport current. The authors used the continuous medium approximation, which is valid in the limit of long wavelength $\lambda_{w}>a$, where $a$ is the intervortex distance. In this limit it does not matter whether the vortices form a regular or disordered array, hence, the effects connected with the periodicity of the vortex field can not be detected.

In the present paper we investigate theoretically the magnon spectrum in the mixed state of a superconducting ferromagnet by solving the phenomenological Landau-Lifshitz and London equations. Vortex motion is taken into account using a viscous damping equation (see Ref. 15). Our analysis extends to the case of short wavelengths $\lambda_{w} \lesssim a$, where Bragg scattering of magnons by the vortex lattice becomes important. The magnon spectrum has a Bloch-like band structure with frequency gaps between adjacent bands. These gaps manifest themselves as anomalies in the frequency dependence of the reflectivity coefficient of electromagnetic radiation.

The outline of the paper is as follows. In Sec. II A we present a model of the ferromagnetic superconductor and review the result for the magnetic excitations spectrum in the Meissner state (see Ref. 8). In Sec. II B we derive the basic equations for the collective vortex-magnetization dynamics. In Secs. IIC and IID the weak-binding approximation is developed and the frequency gaps between adjacent bands of the magnon spectrum are determined analytically. The numerical spectra, obtained using realistic parameters, are presented in Sec. IIE. In Sec. IIF the role of dissipation connected with viscous vortex motion is discussed. Finally, in Sec. III we consider a boundary problem for an electromagnetic wave incident at a ferromagnetic superconductor. The frequency-dependent reflectivity coefficient is examined for frequencies lying within and close to the gaps of the magnon scpectrum.

\section{MAGNON SPECTRUM IN A FERROMAGNETIC SUPERCONDUCTOR IN THE MIXED STATE}

\section{A. Model of the medium and magnetic excitations in the} Meissner state

Let us consider a superconducting ferromagnet with an easy-axis magnetocrystalline anisotropy. At the beginning we assume the exchange interaction and superconducting properties of the material to be isotropic. A generalization 
for the case of uniaxial anisotropy is briefly discussed in the end of Sec. II B.

Within the London approximation the Gibbs free energy of the ferromagnet is ${ }^{6}$

$$
\begin{aligned}
F= & \int\left[\frac{\alpha}{2}\left(\frac{\partial \mathbf{M}}{\partial x_{i}} \frac{\partial \mathbf{M}}{\partial x_{i}}\right)+\frac{K \mathbf{M}_{\perp}^{2}}{2}+\frac{1}{8 \pi \lambda^{2}}\left(\mathbf{A}-\frac{\Phi_{0}}{2 \pi} \nabla \theta\right)^{2}\right. \\
& \left.+\frac{(\operatorname{rot} \mathbf{A}-4 \pi \mathbf{M})^{2}}{8 \pi}-\frac{\mathbf{H}_{e} \operatorname{rot} \mathbf{A}}{4 \pi}\right] d^{3} \mathbf{r}
\end{aligned}
$$

Here $\mathbf{M}$ is the magnetization, $\alpha$ is the exchange constant, $\lambda$ is the London length, $K$ is the anisotropy coefficient, $\Phi_{0}$ is the flux quantum, $\theta$ is the superconducting phase, $\mathbf{H}_{e}$ is an external magnetic field, and $\mathbf{M}_{\perp}$ is the component of $\mathbf{M}$ perpendicular to the anisotropy axis which we direct along the $z$ axis. By setting the variational derivative of $F$ with respect to $\mathbf{A}$ equal to zero we obtain the London equation

$$
\operatorname{rot} \operatorname{rot} \mathbf{B}+\frac{\mathbf{B}}{\lambda^{2}}=4 \pi \operatorname{rot} \operatorname{rot} \mathbf{M}+\frac{1}{\lambda^{2}} \kappa,
$$

where $\mathbf{B}=\operatorname{rot} \mathbf{A}$, and $\boldsymbol{\kappa}$ is the vorticity

$$
\boldsymbol{\kappa}=\frac{\Phi_{0}}{2 \pi} \operatorname{rot} \nabla \theta
$$

It has been assumed that the external field has no sources inside the material. The magnetization vector obeys the LandauLifshitz equation

$$
\frac{\partial \mathbf{M}}{\partial t}=-\gamma \frac{\delta F}{\delta \mathbf{M}} \times \mathbf{M},
$$

or

$$
\frac{\partial \mathbf{M}}{\partial t}=\gamma\left(\alpha \nabla^{2} \mathbf{M}-K \mathbf{M}_{\perp}+\mathbf{B}\right) \times \mathbf{M} .
$$

We will determine the spectrum of low-energy excitations in our system. First, we recall the spectrum for the Meissner state. In equilibrium $\mathbf{A}=0, \mathbf{M}=\mathbf{M}_{0}=M \mathbf{z}_{0}$, and $\boldsymbol{\kappa}=0$. For small perturbations we can linearize Eqs. (2) and (5) with respect to the deviation from equilibrium

$$
\begin{gathered}
\operatorname{rot} \operatorname{rot} \mathbf{B}+\frac{\mathbf{B}}{\lambda^{2}}=4 \pi \operatorname{rot} \operatorname{rot} \mathbf{m}, \\
\frac{\partial \mathbf{m}}{\partial t}=\gamma\left(\alpha \nabla^{2} \mathbf{m}-K \mathbf{m}+\mathbf{B}\right) \times \mathbf{M}_{\mathbf{0}},
\end{gathered}
$$

where $\mathbf{m}=\mathbf{M}_{\perp}$. Assuming $\mathbf{B}, \mathbf{m} \sim e^{-i \omega t+i k_{z} z+i \mathbf{q r}}$, where $\mathbf{q}=$ $\left(q_{x}, q_{y}, 0\right)$, we obtain the magnon spectrum (see Ref. 8)

$$
\begin{gathered}
\omega=\omega_{0}(q)=\gamma M \sqrt{K_{1} K_{2}}, \\
K_{1}\left(q, k_{z}\right)=\tilde{K}+\alpha q^{2}-\frac{4 \pi k_{z}^{2}}{\lambda^{-2}+k_{z}^{2}+q^{2}}, \\
K_{2}\left(q, k_{z}\right)=\tilde{K}+\alpha q^{2}-\frac{4 \pi\left(k_{z}^{2}+q^{2}\right)}{\lambda^{-2}+k_{z}^{2}+q^{2}},
\end{gathered}
$$

where $\tilde{K}=K+\alpha k_{z}^{2}$.

The parameters of some U-based compounds are listed in Table I. It can be seen that these supercondutors
TABLE I. Parameters of ferromagnetic superconductors. The data have been taken from Refs. 1,16-18.

\begin{tabular}{lcccc}
\hline \hline \multicolumn{4}{c}{$\begin{array}{c}\text { Effective } \\
\text { domain wall } \\
\text { thickness, }\end{array}$} & $\begin{array}{c}\text { Anisotropy } \\
\text { field, }\end{array}$ \\
Compound & $\tilde{w} \sim \sqrt{\alpha / K}, \mathrm{~nm}$ & $H_{\text {an }}, \mathrm{T}$ & $K=H_{\text {an }} / M_{0}$ & $\lambda, \mathrm{nm}$ \\
\hline $\mathrm{UGe}_{2}$ & 13,6 & $\sim 100$ & $\sim 10^{4}$ & 1000 \\
$\mathrm{UCoGe}$ & 45 & $\sim 10$ & $\sim 10^{4}$ & 1200 \\
$\mathrm{URhGe}$ & 3450 & $\sim 10$ & $\sim 10^{3}-10^{4}$ & 900 \\
\hline \hline
\end{tabular}

possess a rather high magnetic anisotropy. This fact allows to simplify the expression for the frequency by expanding the root in Eq. (8) in the powers of the small quantity $\left(K_{1}-K_{2}\right) / K_{1}$

$$
\omega_{0}\left(q, k_{z}\right) \approx \gamma M\left[\tilde{K}+\alpha q^{2}-\frac{2 \pi\left(2 k_{z}^{2}+q^{2}\right)}{\lambda^{-2}+k_{z}^{2}+q^{2}}\right] .
$$

A characteristic feature of this spectrum is the presence of a minimum at

$$
q=q_{\min }=\sqrt{\sqrt{2 \pi \frac{\lambda^{-2}-k_{z}^{2}}{\alpha}}-\lambda^{-2}-k_{z}^{2}}
$$

for sufficiently small $k_{z}$. The $\omega_{0}$ vs. $q$ dependence for $k_{z}=0$ is depicted in Fig. 1.

\section{B. Magnetic exitations in the mixed state: Basic equations}

Now we consider a more realistic case of a ferromagnetic superconductor in the mixed state. If the external magnetic field is absent or parallel to the easy axis, the Abrikosov vortices in equilibrium are directed along the magnetization vector. We assume the vortex lattice to be triangular with the positions of the vortices given by the vectors

$$
\mathbf{R}_{i}=a \mathbf{y}_{0} p+a\left(\frac{\sqrt{3}}{2} \mathbf{x}_{0}+\frac{1}{2} \mathbf{y}_{0}\right) n,
$$

where $a$ is the distance between neighboring vortices and $p$ and $n$ are integers. Then, the equilibrium vorticity and magnetic

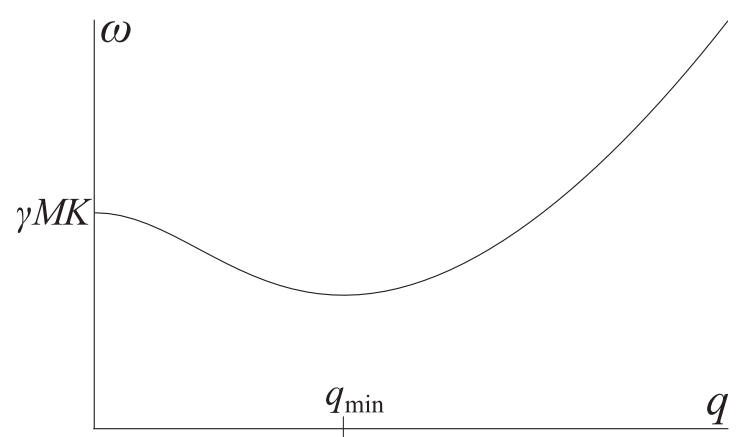

FIG. 1. The magnon spectrum in the Meissner state. 
field are

$$
\begin{gathered}
\boldsymbol{\kappa}_{0}=\Phi_{0} \mathbf{z}_{0} \sum_{i} \delta^{(2)}\left(\boldsymbol{\rho}-\mathbf{R}_{i}\right), \\
\mathbf{B}_{0}=\mathbf{z}_{0} \sum_{G<\xi^{-1}} B_{0}(\mathbf{G}) e^{i \mathbf{G r}}, \quad B_{0}(\mathbf{G})=\frac{\Phi_{0}}{1+G^{2} \lambda^{2}} \cdot \frac{2}{\sqrt{3} a^{2}},
\end{gathered}
$$

where $\rho=(x, y), \xi$ is the coherence length, and $\mathbf{G}$ are the vectors of the reciprocal lattice

$\mathbf{G}=p \mathbf{G}_{1}+n \mathbf{G}_{2}, \quad \mathbf{G}_{1}=\frac{4 \pi}{\sqrt{3} a} \mathbf{x}_{0}, \quad \mathbf{G}_{2}=\frac{2 \pi}{\sqrt{3} a} \mathbf{x}_{0}+\frac{2 \pi}{a} \mathbf{y}_{0}$.

Following $\mathrm{Ng}$ and Varma, ${ }^{15}$ we will consider the magnetization dynamics, taking into account vortex motion as well. The linearized equations (2) and (4) read

$$
\begin{aligned}
\frac{\partial \mathbf{m}}{\partial t}= & \gamma M\left[\alpha \nabla^{2} \mathbf{m}-\left(K+\frac{B_{0}(\mathbf{r})}{M}\right) \mathbf{m}+\mathbf{b}\right] \times \mathbf{z}_{0}, \\
& -\nabla^{2} \mathbf{b}+\frac{\mathbf{b}}{\lambda^{2}}=4 \pi \operatorname{rot} \operatorname{rot} \mathbf{m}+\frac{1}{\lambda^{2}} \kappa_{1},
\end{aligned}
$$

where $\mathbf{b}=\mathbf{B}-\mathbf{B}_{\mathbf{0}}$ and $\boldsymbol{\kappa}_{1}=\boldsymbol{\kappa}-\boldsymbol{\kappa}_{0}$. The linear deviation from equilibrium of the vorticity is given by

$$
\begin{aligned}
\kappa_{1}= & \Phi_{0} \sum_{i}\left\{\delta^{(2)}\left(\rho-\mathbf{R}_{i}\right) \frac{d \Delta \mathbf{R}_{i}}{d z}\right. \\
& \left.-\mathbf{z}_{0}\left[\Delta \mathbf{R}_{i} \cdot \nabla_{\rho} \delta^{(2)}\left(\rho-\mathbf{R}_{i}\right)\right]\right\},
\end{aligned}
$$

where $\Delta \mathbf{R}_{i}(z)$ is the local displacement of the vortex with respect to its equilibrium position $\mathbf{R}_{i}$. To determine the quantities $\Delta \mathbf{R}_{i}$ we use the phenomonological equation of dissipative vortex dynamics ${ }^{15}$

$$
\eta \frac{d}{d t} \Delta \mathbf{R}_{i}(z)=-\frac{\delta F}{\delta \Delta \mathbf{R}_{i}},
$$

where $\eta$ is a viscosity coefficient. After the evaluation of the variational derivative, Eq. (20) transforms into

$$
\begin{aligned}
-\eta & \frac{d}{d t} \Delta \mathbf{R}_{i}(z) \\
= & \frac{\Phi_{0}}{(2 \pi)^{3 / 2}} \int_{k<\xi^{-1}} \frac{-i k_{z}\left(\boldsymbol{\kappa}_{1 k}-4 \pi \mathbf{m}_{k}\right)+i \mathbf{k}\left(\mathbf{z}_{0} \boldsymbol{\kappa}_{1 k}\right)}{4 \pi\left(\lambda^{2} k^{2}+1\right)} \\
& \times \exp \left(i \mathbf{k} \mathbf{R}_{i}+i k_{z} z\right) d^{3} \mathbf{k}-\sum_{G<\xi^{-1}} \frac{B_{0}(0) \Phi_{0}\left(\mathbf{G} \Delta \mathbf{R}_{i}\right) \mathbf{G}}{4 \pi\left(\lambda^{2} G^{2}+1\right)}
\end{aligned}
$$

where $\xi$ is the superconducting coherence length, and $X_{k}$ for any quantity $X$ denotes its Fourier transform

$$
X_{k}=\frac{1}{(2 \pi)^{3 / 2}} \int X(\mathbf{r}) e^{-i \mathbf{k r}} d^{3} \mathbf{r} .
$$

We may rewrite Eqs. (17), (18), and (21) in the Fourier representation. If we do so, we will find that these equations connect the Fourier components of the functions $\mathbf{m}, \mathbf{b}$, and $\boldsymbol{\kappa}$ corresponding to wave vectors satisfying the condition

$$
\mathbf{k}=\mathbf{G}+\mathbf{k}_{0},
$$

where $\mathbf{k}_{0}$ is a fixed arbitrary vector and the vector $\mathbf{G}$ runs over the whole reciprocal lattice (16). Hence, the general solution of Eqs. (17), (18), and (21) can be presented as a superposition of particular solutions having the form

$$
\begin{gathered}
\mathbf{m}=e^{-i \omega t+i k_{z} z+i \mathbf{q r}} \sum_{\mathbf{G}} \mathbf{m}(\mathbf{G}) e^{i \mathbf{G r}}, \\
\mathbf{b}=e^{-i \omega t+i k_{z} z+i \mathbf{q r}} \sum_{\mathbf{G}} \mathbf{b}(\mathbf{G}) e^{i \mathbf{G r}}, \\
\boldsymbol{\kappa}_{1}=e^{-i \omega t+i k_{z} z+i \mathbf{q r}} \sum_{\mathbf{G}} \boldsymbol{\kappa}_{1}(\mathbf{G}) e^{i \mathbf{G r}},
\end{gathered}
$$

where $\mathbf{q}$ is the quasi-wave-vector in the $x y$ plane. The fact that the functions (23) to (25) satisfy our equations represents a simple generalization of the Bloch theorem. The condition (25) is equivalent to the following one:

$$
\Delta \mathbf{R}_{i}(z)=\Delta \mathbf{R} e^{-i \omega t+i k_{z} z+i \mathbf{q} \mathbf{R}_{i}} .
$$

If we substitute Eqs. (23), (24), and (26) into Eqs. (17), (18), and (21), we obtain the system

$$
\begin{aligned}
& \frac{i \omega \eta}{\Phi_{0}} \Delta \mathbf{R}=\sum_{G_{i}<\xi^{-1}} \frac{4 \pi i k_{z} \mathbf{m}\left(\mathbf{G}_{i}\right)+B_{0}(0) k_{z}^{2} \Delta \mathbf{R}}{4 \pi\left[1+\lambda^{2}\left(k_{z}^{2}+q_{i}^{2}\right)\right]}+\sum_{G_{i}<\xi^{-1}} \frac{B_{0}(0)}{4 \pi}\left[\frac{\mathbf{q}_{i}\left(\mathbf{q}_{i} \Delta \mathbf{R}\right)}{1+\lambda^{2}\left(q_{i}^{2}+k_{z}^{2}\right)}-\frac{\mathbf{G}(\mathbf{G} \Delta \mathbf{R})}{1+\lambda^{2} G^{2}}\right], \\
&-\frac{i \omega}{\gamma M} \mathbf{m}\left(\mathbf{G}_{i}\right)= {\left[-\left(\tilde{K}+\alpha q_{i}^{2}\right) \mathbf{m}\left(\mathbf{G}_{i}\right)+\frac{4 \pi\left(k_{z}^{2}+q_{i}^{2}\right) \mathbf{m}\left(\mathbf{G}_{i}\right)-4 \pi \mathbf{q}_{i}\left(\mathbf{q}_{i} \mathbf{m}\left(\mathbf{G}_{i}\right)\right)+B_{0}(0) \lambda^{-2} i k_{z} \Delta \mathbf{R}}{k_{z}^{2}+q_{i}^{2}+\lambda^{-2}}\right.} \\
&\left.-\frac{1}{M} \sum_{\mathbf{G}^{\prime} \neq \mathbf{G}_{i}} \mathbf{m}\left(\mathbf{G}^{\prime}\right) B_{0}\left(\mathbf{G}_{i}-\mathbf{G}^{\prime}\right)\right] \times \mathbf{z}_{0},
\end{aligned}
$$

where $\mathbf{q}_{i}=\mathbf{q}+\mathbf{G}_{i}$, and

$$
\tilde{K}=K+B_{0}(0) / M+\alpha k_{z}^{2} .
$$

By solving Eqs. (27) and (28) the dispersion relation may be found.
First, we restrict ourselves to the case when the dissipation due to vortex motion is negligible, i.e., $\eta \rightarrow \infty$ and $\Delta \mathbf{R}=0$. The role of thermal losses will be discussed in Sec. II F.

It has been mentioned that in the U-based compounds the magnetic anisotropy is rather large. Using this fact 
one can make an approximation which will considerably simplify the problem. Let the vectors $\mathbf{q}_{i}$ have the components $\left(q_{i} \cos \alpha_{i}, q_{i} \sin \alpha_{i}\right)$. For $q_{i}=0$ the angle $\alpha_{i}$ is arbitrary. We introduce the new variables

$$
\begin{aligned}
& m_{i x}^{\prime}=\left[\cos \alpha_{i} m_{x}\left(\mathbf{G}_{i}\right)+\sin \alpha_{i} m_{y}\left(\mathbf{G}_{i}\right)\right] \sqrt[4]{\frac{K_{1}\left(q_{i}\right)}{K_{2}\left(q_{i}\right)}}, \\
& m_{i y}^{\prime}=\left[\cos \alpha_{i} m_{y}\left(\mathbf{G}_{i}\right)-\sin \alpha_{i} m_{x}\left(\mathbf{G}_{i}\right)\right] \sqrt[4]{\frac{K_{2}\left(q_{i}\right)}{K_{1}\left(q_{i}\right)}},
\end{aligned}
$$

where $K_{1}$ and $K_{2}$ are given by Eqs. (9) and (10). Here and further we omit $k_{z}$ in the list of arguments of $K_{1}, K_{2}$, and $\omega_{0}$ for brevity. The quantities $m_{i x}^{\prime}$ and $m_{i y}^{\prime}$ satisfy the equations

$$
\begin{aligned}
-\frac{i \omega}{\gamma M} m_{i x}^{\prime}= & -\frac{\omega_{0}\left(q_{i}\right)}{\gamma M} m_{i y}^{\prime}-\sum_{j \neq i} b_{i j}\left[m_{j y}^{\prime} \sqrt[4]{\frac{K_{1}\left(q_{i}\right) K_{1}\left(q_{j}\right)}{K_{2}\left(q_{i}\right) K_{2}\left(q_{j}\right)}}\right. \\
& \times \cos \left(\alpha_{i}-\alpha_{j}\right)-m_{j x}^{\prime} \sqrt[4]{\frac{K_{1}\left(q_{i}\right) K_{2}\left(q_{j}\right)}{K_{2}\left(q_{i}\right) K_{1}\left(q_{j}\right)}} \\
& \left.\times \sin \left(\alpha_{i}-\alpha_{j}\right)\right], \\
-\frac{i \omega}{\gamma M} m_{i y}^{\prime}= & \frac{\omega_{0}\left(q_{i}\right)}{\gamma M} m_{i x}^{\prime}+\sum_{j \neq i} b_{i j}\left[m_{j x}^{\prime} \sqrt[4]{\frac{K_{2}\left(q_{i}\right) K_{2}\left(q_{j}\right)}{K_{1}\left(q_{i}\right) K_{1}\left(q_{j}\right)}}\right. \\
& \times \cos \left(\alpha_{i}-\alpha_{j}\right)+m_{j y}^{\prime} \sqrt[4]{\frac{K_{2}\left(q_{i}\right) K_{1}\left(q_{j}\right)}{K_{1}\left(q_{i}\right) K_{2}\left(q_{j}\right)}} \\
& \left.\times \sin \left(\alpha_{i}-\alpha_{j}\right)\right],
\end{aligned}
$$

where $b_{i j}=B_{0}\left(\mathbf{G}_{i}-\mathbf{G}_{j}\right) / M$, and

$$
\frac{\omega_{0}(q)}{\gamma M} \approx K+\alpha\left(q^{2}+k_{z}^{2}\right)+\frac{B_{0}(0)}{M}-\frac{2 \pi\left(2 k_{z}^{2}+q^{2}\right)}{\lambda^{-2}+k_{z}^{2}+q^{2}} .
$$

The main assumption of our approximation is that all fourth roots in Eqs. (30) and (31) can be replaced by unity. Indeed,

$$
\begin{aligned}
& \sqrt[4]{\frac{K_{1}\left(q_{i}\right) K_{1}\left(q_{j}\right)}{K_{2}\left(q_{i}\right) K_{2}\left(q_{j}\right)}-1} \\
& \approx \frac{1}{4}\left[\frac{K_{1}\left(q_{i}\right)-K_{2}\left(q_{i}\right)}{K_{2}\left(q_{i}\right)}+\frac{K_{1}\left(q_{j}\right)-K_{2}\left(q_{j}\right)}{K_{2}\left(q_{j}\right)}\right] \ll 1 .
\end{aligned}
$$

It is convenient to introduce the variables $m_{i}^{+}=\left(m_{i x}^{\prime}-\right.$ $\left.i m_{i y}^{\prime}\right) e^{-i \alpha_{i}}$ and $m_{i}^{-}=\left(m_{i x}^{\prime}+i m_{i y}^{\prime}\right) e^{i \alpha_{i}}$. Equations (30) and (31) yield

$$
\begin{gathered}
\frac{\omega}{\gamma M} m_{i}^{+}=\frac{\omega_{0}\left(q_{i}\right)}{\gamma M} m_{i}^{+}+\sum_{j \neq i} b_{i j} m_{j}^{+}, \\
-\frac{\omega}{\gamma M} m_{i}^{-}=\frac{\omega_{0}\left(q_{i}\right)}{\gamma M} m_{i}^{-}+\sum_{j \neq i} b_{i j} m_{j} .
\end{gathered}
$$

It can be seen that the solutions of Eq. (34) coincide with those of Eq. (33), but the frequencies have the opposite sign. A great advantage of Eq. (33) over Eq. (28) is that it represents an eigenvalue problem for a real symmetric matrix, so simple numerical and analytical procedures may be applied to solve it.
Equation (33) can be also derived if the uniaxial $(z$ axis) superconducting and exchange interaction anisotropy is taken into account. The only modification is that the Fourier components of the field and the unperturbed frequencies are given by

$$
\begin{gathered}
B_{0}(\mathbf{G})=\frac{\Phi_{0}}{1+G^{2} \lambda_{\perp}^{2}} \cdot \frac{2}{\sqrt{3} a^{2}}, \\
\frac{\omega_{0}(q)}{\gamma M} \approx K+\alpha_{\|} k_{z}^{2}+\alpha_{\perp} q^{2}+\frac{B_{0}(0)}{M} \\
-\frac{2 \pi k_{z}^{2} \lambda_{\perp}^{2}}{1+\lambda_{\perp}^{2}\left(k_{z}^{2}+q^{2}\right)}-\frac{2 \pi\left(k_{z}^{2} \lambda_{\perp}^{2}+q^{2} \lambda_{\|}^{2}\right)}{1+k_{z}^{2} \lambda_{\perp}^{2}+q^{2} \lambda_{\|}^{2}},
\end{gathered}
$$

where the quantities $\alpha_{\|}, \lambda_{\|}$are related to the $z$ axis, and $\alpha_{\perp}$, $\lambda_{\perp}$ are related to the perpendicular plane.

\section{Weak-binding approximation}

To determine the eigenvalues of the system (33) we are going to use a method, which is equivalent to the weak-binding approximation for electrons in a crystal. We assume that several eigenvalues $\omega$ for a given vector $\mathbf{q}$ are close to $\omega_{0}(q)$, and the deviations $\omega-\omega_{0}(q)$ can be determined using the degenerate state perturbation theory.

Let us find the applicability conditions for this approximation. Consider the case when $\omega \approx \omega_{0}\left(q_{i}\right),\left|m_{i}^{+}\right| \geqslant\left|m_{j}^{+}\right|$and all the quantities $\omega_{0}\left(q_{j}\right)$ are not close to $\omega_{0}\left(q_{i}\right)$ for $j \neq i$. Then the perturbation theory in its simplest form can be applied. It follows from Eq. (33) that for $i \neq j$

$$
m_{j} \approx \frac{b_{i j} m_{i} \gamma M}{\omega_{0}\left(q_{i}\right)-\omega_{0}\left(q_{j}\right)} .
$$

Here and further we omit the upper index "+ " for brevity. The correction to the unperturbed frequency $\omega_{0}\left(q_{i}\right)$ due to the fact that $m_{j} \neq 0$ equals

$$
\delta \omega=\frac{b_{i j}^{2} \gamma^{2} M^{2}}{\omega_{0}\left(q_{i}\right)-\omega_{0}\left(q_{j}\right)} .
$$

For the perturbation theory to be valid we have to demand at least $|\delta \omega| \ll\left|\omega_{0}\left(q_{i}\right)-\omega_{0}\left(q_{j}\right)\right|$, or

$$
\left\{\frac{b_{i j}}{\left(q_{i}-q_{j}\right)\left(q_{i}+q_{j}\right)\left[\alpha-\frac{2 \pi \lambda^{-2}}{\left(\lambda^{-2}+q_{i}^{2}\right)\left(\lambda^{-2}+q_{j}^{2}\right)}\right]}\right\}^{2} \ll 1
$$

for all $j \neq i$. Here we assumed $k_{z}=0$ for simplicity.

If we apply the perturbation theory for a degenerate state, we can permit the condition (37) to be violated for $N_{j}>1$ different indices $j$. The number $N_{j}$ can be estimated as

$$
N_{j} \sim S_{q} a^{2},
$$

where $S_{q}$ is the area in the $q$ plane occupied by the vectors $q_{j}$ for which the condition

$$
\frac{b_{i j}}{\left|q_{i}-q_{j}\right|\left(q_{i}+q_{j}\right)\left|\alpha-\frac{2 \pi \lambda^{-2}}{\left(\lambda^{-2}+q_{i}^{2}\right)\left(\lambda^{-2}+q_{j}^{2}\right)}\right|} \geqslant 1
$$

holds. To avoid solving secular equations for large matrices, we demand $N_{q} \sim 1$. Hence, the area $S_{q}$ should not be too large. Restrictions on $S_{q}$ are the most strong in two cases:

(i) $q_{i}$ is close to the value corresponding to the minimum of 
$\omega_{0}\left(q_{i}\right)$ and (ii) $q_{i}$ is sufficiently large. To estimate $S_{q}$ in the first case, we take $q_{i}$ equal to $q_{\text {min }}$. Assuming $\alpha \sim \lambda^{2}$ (which seems to be realistic, according to Table I), $q_{\min } \sim \lambda^{-1}$ and $b_{i j} \sim \Phi_{0} /\left(a^{2} M\right)$ we obtain from Eq. (39)

$$
\left|q-q_{\min }\right| \lesssim \frac{1}{a} \sqrt{\frac{\Phi_{0}}{\lambda^{2} M}}
$$

Hence,

$$
S_{q} \approx 4 \pi q_{\min } \frac{1}{a} \sqrt{\frac{\Phi_{0}}{\lambda^{2} M}},
$$

and

$$
N_{q} \sim \frac{a}{\lambda} \sqrt{\frac{\Phi_{0}}{\lambda^{2} M}} .
$$

Since $N_{q}$ should be of the order of unity, we have the limitation

$$
a \lesssim \lambda \sqrt{\frac{\lambda^{2} M}{\Phi_{0}}} .
$$

Typically, the ratio $\lambda^{2} M / \Phi_{0}$ is not very large. Hence, Eq. (41) means that the intervortex distance should be not much larger than the London length. In further consideration we will imply that the inequality (41) holds.

At the large $q_{i}$ limit we obtain the constraint $\Phi_{0} / M \alpha \lesssim 1$, which is satisfied for the materials listed in Table I.

Similar to the electron spectrum in solid matter, the magnon spectrum in our system consists of bands separated by the gaps. We will use the reduced zone scheme, i.e., the bands are folded in the first Brillouin zone (see Fig. 2).

Now we calculate the gap between two neighboring bands using the weak-binding approximation. Obviously, the gap is the smallest on the lines of the $q$ plane where the bands would intersect if the matrix elements $b_{i j}$ were negligible. On these lines $\omega \approx \omega_{0}\left(q_{i}\right)=\omega_{0}\left(q_{j}\right)$ for some different indices $i$ and $j$. To find small corrections to the unperturbed frequency $\omega_{0}\left(q_{i}\right)$

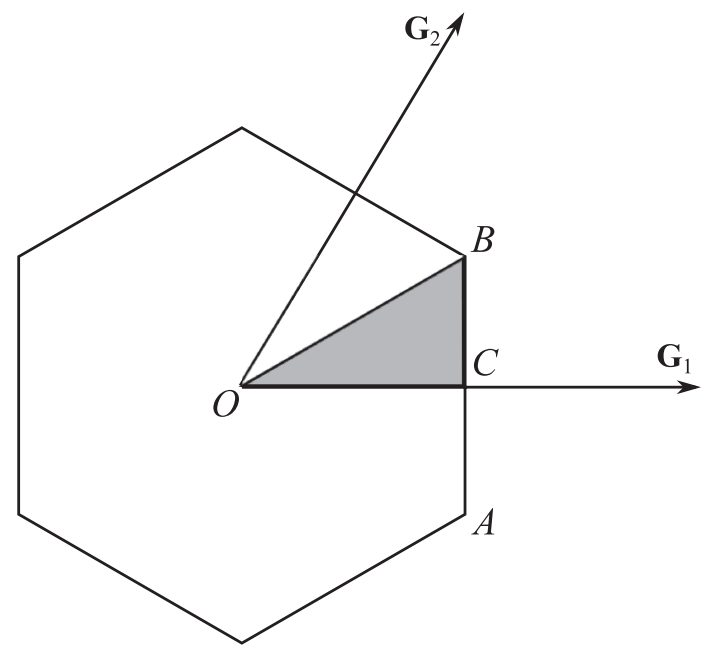

FIG. 2. First Brillouin zone. It is sufficient to calculate the spectrum in the shaded area to determine the spectrum in the whole zone using symmetry relations. one should solve the simple secular equation

$$
\left|\begin{array}{cc}
\frac{\omega_{0}\left(q_{i}\right)-\omega}{\gamma M} & b_{i j} \\
b_{i j} & \frac{\omega_{0}\left(q_{i}\right)-\omega}{\gamma M}
\end{array}\right|=0 .
$$

The frequency gap $\Delta$ is

$$
\Delta=2 \gamma B_{0}\left(\mathbf{G}_{i}-\mathbf{G}_{j}\right) .
$$

It is remarkable that the gap does not change along the line where the two bands would intersect, if $B_{0}\left(\mathbf{G}_{i}-\mathbf{G}_{j}\right)$ was equal to zero.

The dimensionless parameter characterizing the modification of the unperturbed spectrum (11) by the vortex lattice is the ration of the gap $\Delta$ to the frequency. For the compounds listed in Table I this ratio is the largest in URhGe, where

$$
\frac{\Delta}{\omega} \sim \frac{\Phi_{0}}{\lambda^{2} H_{\mathrm{an}}} \sim 10^{-2}-10^{-3} .
$$

This parameter appears to be small due to the large magnetic anisotropy of the compound. According to Eq. (44), the effects connected with the band structure of the magnon spectrum will be more pronounced in materials with low anisotropy.

\section{Magnon spectrum in points of high symmetry: Intersections of bands}

In previous sections we made calculations assuming that the ferromagnet has isotropic superconducting properties, but the results derived there are also qualitatively valid for anisotropic (uniaxial and biaxial) superconductors. In this section we consider the properties of the band structure which appear only in materials with uniaxial symmetry.

We will study the magnon spectrum in points of the Brillouin zone where the system (33) admits a nontrivial symmetry group. We restrict ourself to the case of a relatively strong magnetic field, when $\omega_{0}\left(q_{i}\right)=\omega_{0}\left(q_{j}\right)$ implies $q_{i}=q_{j}$, i.e., the nonmonontonicity of the unperturbed spectrum (36) is irrelevant.

The point with the highest symmetry is the center of the Brillouin zone, where $\mathbf{q}=0$. The corresponding symmetry group is

$$
\mathcal{G}_{0}=\left\{R^{i}, \sigma_{i}\right\}, \quad i=0 . .5,
$$

which is isomorphic to the group $C_{6 v}$. Here $\sigma_{i}=R^{i} \sigma_{0}$, and $R$ and $\sigma_{0}$ are defined as follows:

$$
\begin{aligned}
R: \quad m_{i} & =m\left(\mathbf{G}_{i}\right) \rightarrow m\left(\hat{T} \mathbf{G}_{i}\right), \\
\hat{T} & =\left(\begin{array}{cc}
\cos \frac{\pi}{3} & -\sin \frac{\pi}{3} \\
\sin \frac{\pi}{3} & \cos \frac{\pi}{3}
\end{array}\right) ; \\
\sigma_{0}: \quad m_{i} & =m\left(G_{i x}, G_{i y}\right) \rightarrow m\left(G_{i x},-G_{i y}\right) .
\end{aligned}
$$

The characters for the irreducible representations of the group $\mathcal{G}_{0}$ are listed in Table II. Due to the presence of the two-dimensional representations $B_{1}$ and $B_{2}$ intersections of two bands appear in the center of the Brillouin zone. Indeed, consider the bands with the numbers from 2 to 7 . In the zero-order perturbation theory $\omega(\mathbf{q}=0)=\omega_{0}\left(\mathbf{G}_{1}\right)$ in all these bands. In the first-order perturbation theory we have to take into account the six components $m\left(\hat{T}^{i} \mathbf{G}_{1}\right), i=0 . .5$, where the matrix $\hat{T}$ has been introduced in Eq. (46). The solutions of the sixth-order eigenvalue problem can be found 
TABLE II. Character table of the group $\mathcal{G}_{0}$.

\begin{tabular}{ccccccc}
\hline \hline & $R^{0}$ & $R, R^{5}$ & $R^{2}, R^{4}$ & $R^{3}$ & $\sigma_{0}, \sigma_{2}, \sigma_{4}$ & $\sigma_{1}, \sigma_{3}, \sigma_{5}$ \\
\hline$A_{1}$ & 1 & 1 & 1 & 1 & 1 & 1 \\
$A_{2}$ & 1 & 1 & 1 & 1 & -1 & -1 \\
$A_{3}$ & 1 & -1 & 1 & -1 & 1 & -1 \\
$A_{4}$ & 1 & -1 & 1 & -1 & -1 & 1 \\
$B_{1}$ & 2 & -1 & -1 & 2 & 0 & 0 \\
$B_{2}$ & 2 & 1 & -1 & -2 & 0 & 0 \\
\hline \hline
\end{tabular}

in Table III. Here $b_{12}=B_{0}\left(\mathbf{G}_{1}\right) / M, b_{13}=B_{0}\left(\mathbf{G}_{1}+\mathbf{G}_{2}\right) / M$, $b_{14}=B_{0}\left(2 \mathbf{G}_{1}\right) / M$. The pairs of bands 3,4 and 5,6 have a point of contact at $\mathbf{q}=0$.

Another point of high symmetry is the $B$ point (see Fig. 2), where $\mathbf{q}=\mathbf{q}_{B}=\left(\mathbf{G}_{1}+\mathbf{G}_{2}\right) / 3$. The corresponding symmetry group is

$$
\mathcal{G}_{B}=\left\{R_{B}^{i}, \sigma_{B i}\right\}, \quad i=0,1,2,
$$

where

$$
\begin{aligned}
R_{B}: \quad m\left(\mathbf{G}_{i}\right) & \rightarrow m\left(\hat{T}^{2} \mathbf{G}_{i}-\mathbf{G}_{1}\right), \\
\sigma_{B 0}: \quad m\left(\mathbf{G}_{i}\right) & \rightarrow m\left(\hat{\sigma}_{O B} \mathbf{G}_{i}\right), \\
\sigma_{B i} & =R_{B}^{i} \sigma_{B 0},
\end{aligned}
$$

and $\hat{\sigma}_{O B}$ is the reflection operator with respect to the $O B$ axis. The group $\mathcal{G}_{B}$ is isomorphic to the group $C_{3 v}$. The characters of its irreducible representations are listed in Table IV.

The intersection of bands in the $B$ point occurs at frequencies close to $\omega_{0}\left(q_{B}\right)$. In the first-order perturbation theory we take into account the elements $m(0), m\left(-\mathbf{G}_{1}\right)$, and $m\left(-\mathbf{G}_{2}\right)$ in Eq. (33). The solutions of the eigenvalue problem are given in Table V. At this time, the first and second band have a point of contact.

Finally, we want to make a remark concerning the symmetry of the initial system (26) to (28). In Sec. II B we derived the approximate equation (33) using the fact that the quantity $\tilde{K}$ is typically very large. As a by-product of this simplification we gained the reflection symmetry operations $\sigma_{i}$ and $\sigma_{B i}$, which are not present in the initial system (to be more accurate, the analogs of $\sigma_{i}$ and $\sigma_{B i}$ involve complex conjugation, so these operations are not linear). The system (26) to (28) for $\mathbf{q}=0$ and $\mathbf{q}=\mathbf{q}_{B}$ admits symmetry groups which are isomorphic to the groups $C_{6}$ and $C_{3}$, respectively, which have only one-dimensional irreducible representations. As a result, a small gap exists between the the bands which had a point of contact within Eq. (33). However, this gap is negligible $\left(\Delta \sim \gamma M b_{i j} / \tilde{K}\right)$ for materials with large magnetocrystalline anisotropy, or at large average magnetic fields $B_{0}(0)$.

TABLE III. The solutions of Eq. (33) with $q=0$ in the first-order perturbation theory.

\begin{tabular}{lccc}
\hline \hline $\begin{array}{l}\text { Represen- } \\
\text { tation }\end{array}$ & $\begin{array}{c}\text { Band } \\
\text { numbers }\end{array}$ & $\begin{array}{c}\text { Solutions } \\
m\left(\hat{T}^{k} \mathbf{G}_{1}\right)=\ldots\end{array}$ & $\frac{\omega-\omega_{0}\left(\mathbf{G}_{1}\right)}{\gamma M}$ \\
\hline$A_{3}$ & 2 & $(-1)^{k} m\left(\mathbf{G}_{1}\right)$ & $-2 b_{12}+2 b_{13}-b_{14}$ \\
$B_{1}$ & 3,4 & $e^{ \pm 2 i k \pi / 3} m\left(\mathbf{G}_{1}\right)$ & $-b_{12}-b_{13}+b_{14}$ \\
$B_{2}$ & 5,6 & $e^{ \pm i k \pi / 3} m\left(\mathbf{G}_{1}\right)$ & $b_{12}-b_{13}-b_{14}$ \\
$A_{1}$ & 7 & $m\left(\mathbf{G}_{1}\right)$ & $2 b_{12}+2 b_{13}+b_{14}$ \\
\hline \hline
\end{tabular}

TABLE IV. Character table of the group $\mathcal{G}_{B}$.

\begin{tabular}{lccc}
\hline \hline & $R_{B}^{0}$ & $R_{B}, R_{B}^{2}$ & $\sigma_{B i}, i=0,1,2$ \\
\hline$D_{1}$ & 1 & 1 & 1 \\
$D_{2}$ & 1 & 1 & -1 \\
$E$ & 2 & -1 & 0 \\
\hline \hline
\end{tabular}

\section{E. Numerical calculation of the magnon spectra}

In this section we give numerical magnon spectra for different average magnetic fields $B_{0}(0)$. We solved Eq. (33) numerically neglecting all Fourier components $m_{i}$ except for those corresponding to the 31 vectors $\mathbf{G}_{i}$ with the smallest lengths. In the weak-binding approximations, this is sufficient to calculate the spectra in the six lowest frequency bands. As the parameters we used those of $\mathrm{UGe}_{2}$ (Refs. 1 and 16): $\lambda=1 \mu \mathrm{m}, \alpha=\left(13.6 \times 10^{-5}\right)^{2} \mathrm{~cm}^{2}, K=10^{4}$, and $M=$ $150 \mathrm{emu} / \mathrm{cm}^{3}$. In all calculations $k_{z}=0$ is assumed.

In Fig. 3 we depict the magnon spectra in the lowest three bands for the average magnetic field equal to

$$
\beta=\frac{\Phi_{0} \sqrt{3}}{2 \pi^{2}}\left(\frac{2 \pi}{\alpha}-\lambda^{-2}\right) .
$$

At this field $\omega_{0}(0)=\omega_{0}\left(G_{1} / 2\right)$. It may be seen that the spectra in the second and third bands have corners. These corners correspond to band intersection lines in zero-order perturbation theory. In fact, the corners are smoothed out, but this may be visible only on a small-scale graph. In Fig. 4 two cross sections of the six lowest bands are shown. In the vertical axis label $\omega_{\mathrm{FM}}=\gamma M K$ is the ferromagnetic resonance frequency. The gaps between some bands are so small that these bands are indistinguishable on the graphs, so they are represented by one curve.

In Figs. 5 to 8 the same spectra for lower magnetic fields are shown. As the field decreases, the shape of the band structure changes qualitatively. For example, at the field $0.25 \beta$ the smooth maximum in the center of the first band transforms into a peak [see Fig. 7(a)]. This peculiar behavior the first band is a consequence of the nonmonotonicity of the unperturbed spectrum (11) and is connected with a commensurability effect: The peak appears when $\omega_{0}(0)=\omega_{0}\left(\mathbf{G}_{1}\right)$.

\section{F. Taking into account dissipation}

In this section we will discuss how the magnon spectrum is modified when dissipation is taken into account. First, we

TABLE V. The solutions of Eq.(33) with $\mathbf{q}=\mathbf{q}_{B}$ in the first-order perturbation theory.

\begin{tabular}{lccc}
\hline \hline $\begin{array}{l}\text { Represen- } \\
\text { tation }\end{array}$ & $\begin{array}{c}\text { Band } \\
\text { numbers }\end{array}$ & Solutions & $\frac{\omega-\omega_{0}\left(\mathbf{q}_{B}\right)}{\gamma M}$ \\
\hline$E$ & 1,2 & $\begin{array}{l}m\left(-\mathbf{G}_{1}\right)=e^{ \pm 2 \pi i / 3} m(0), \\
m\left(-\mathbf{G}_{2}\right)=e^{\mp 2 \pi i / 3} m(0)\end{array}$ & $-b_{12}$ \\
& 3 & $m\left(-\mathbf{G}_{1}\right)=m\left(-\mathbf{G}_{2}\right)=m(0)$ & $2 b_{12}$ \\
\hline$D_{1}$ & 3 &
\end{tabular}




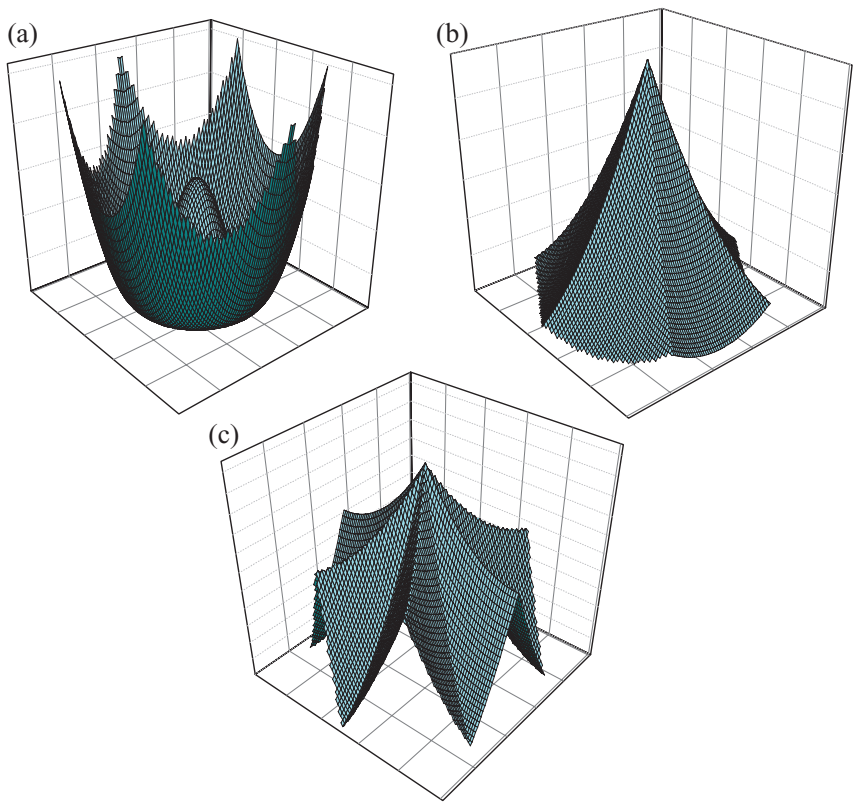

FIG. 3. (Color online) The magnon spectra in the (a) first, (b) second, and (c) third bands. $B_{0}(0)=\beta$.

consider losses due to vortex motion. Generally, it is rather difficult to express the displacement amplitude $\Delta \mathbf{R}$ in terms of $\mathbf{m}\left(\mathbf{G}_{i}\right)$, using Eq. (27). However, a simplification is possible
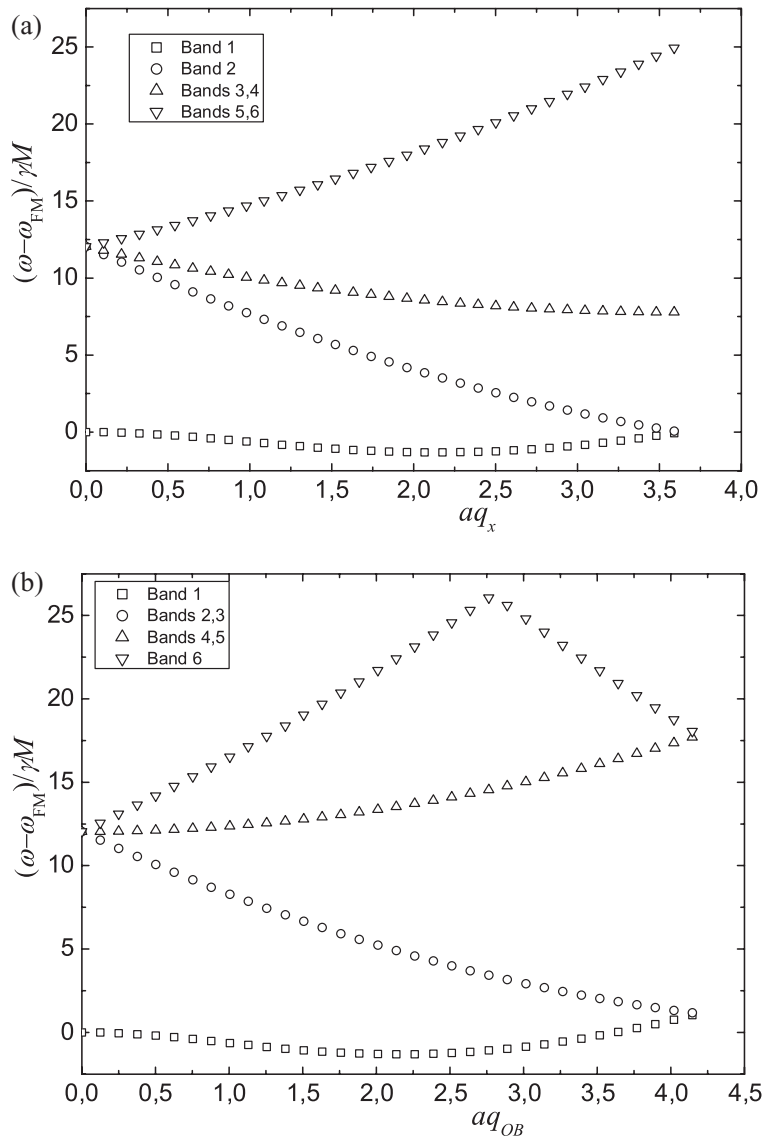

FIG. 4. The cross sections of the lowest six bands along the lines (a) $O C$ and (b) $O B \cdot B_{0}(0)=\beta$.

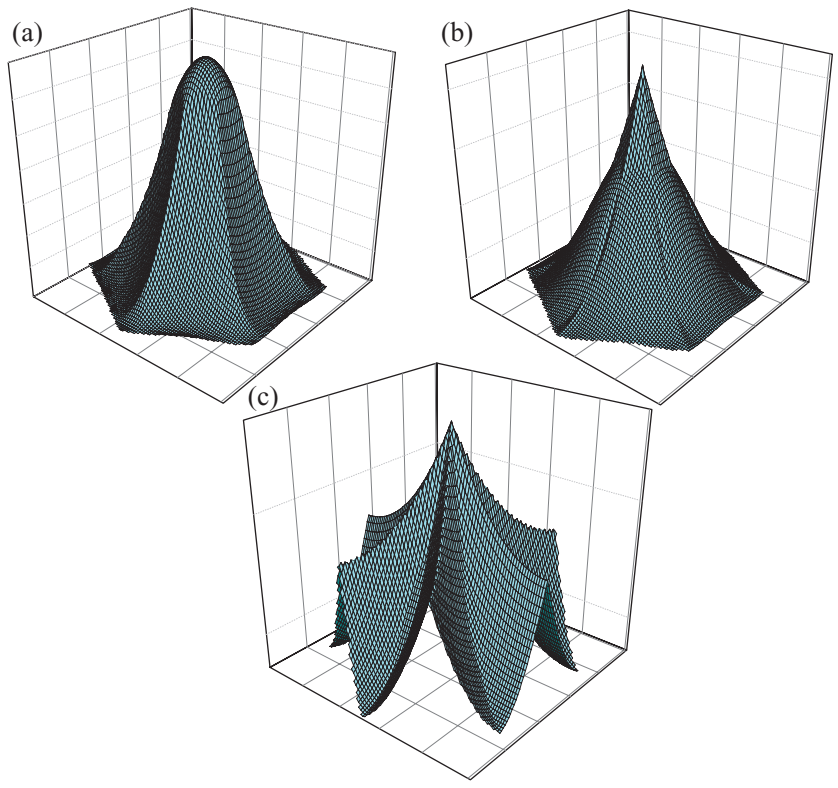

FIG. 5. (Color online) The magnon spectra in the (a) first, (b) second, and (c) third bands. $B_{0}(0)=0.3 \beta$.

in the high- $\eta$ limit, when all terms in the right-hand side of Eq. (27) containing $\Delta \mathbf{R}$ can be neglected as compared to the relaxational term in the left-hand side. For this approximation
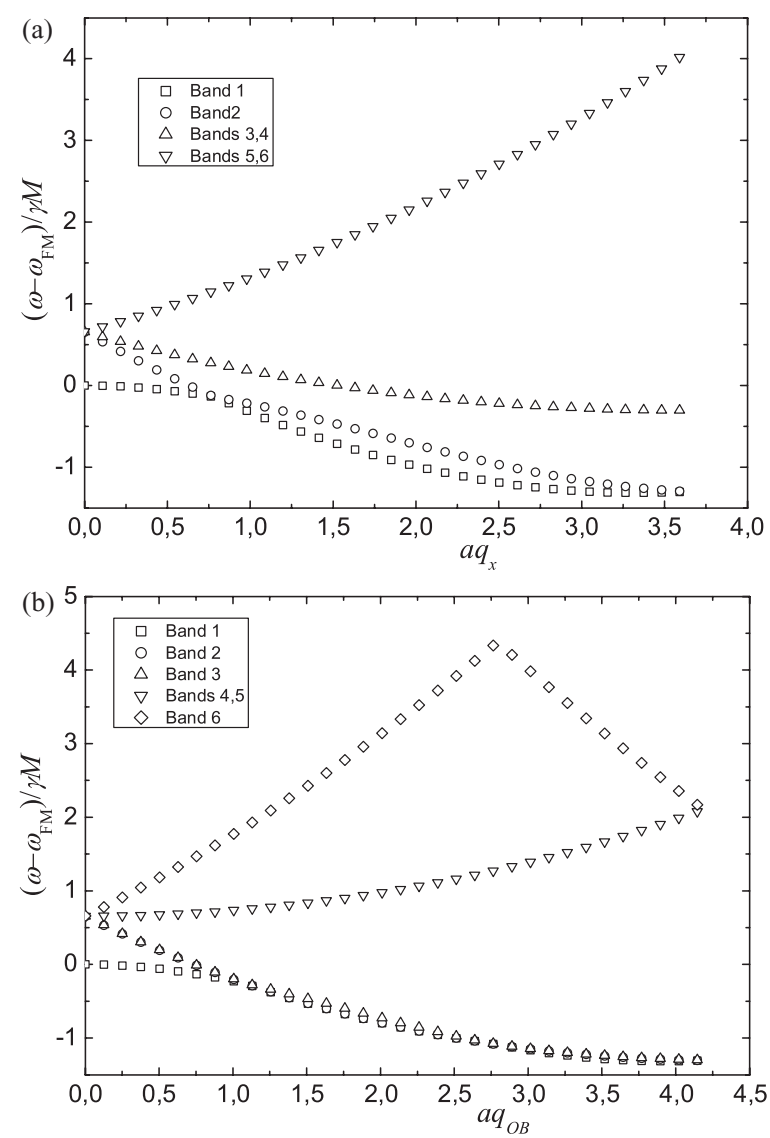

FIG. 6. The cross sections of the lowest six bands along the lines (a) $O C$ and (b) $O B \cdot B_{0}(0)=0.3 \beta$. 


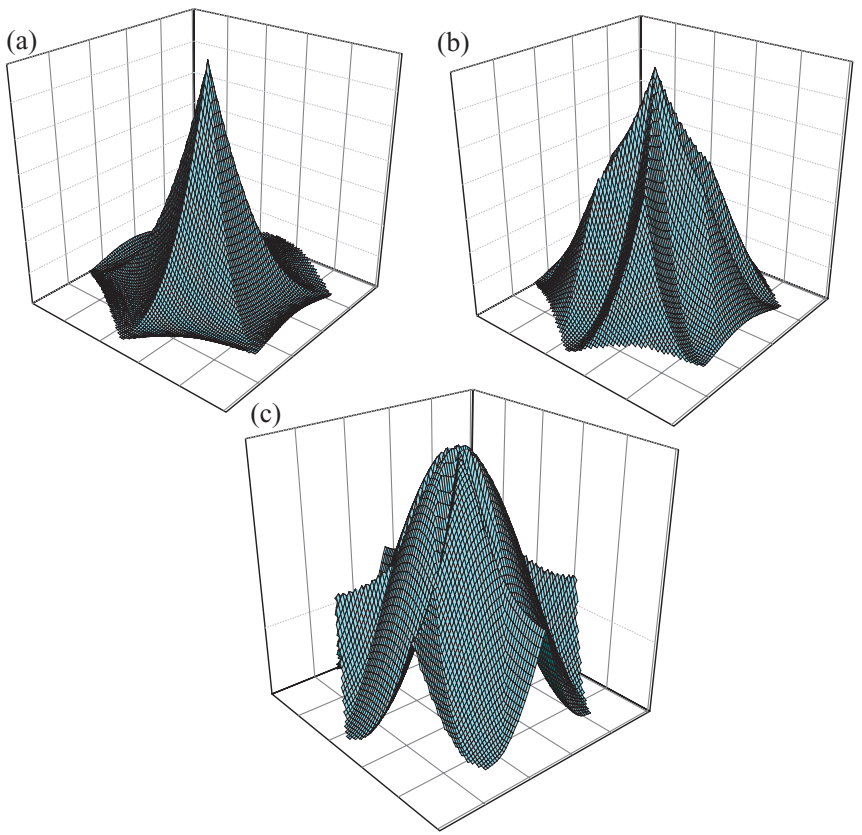

FIG. 7. (Color online) The magnon spectra in the (a) first, (b) second, and (c) third bands. $B_{0}(0)=0.25 \beta$.

to be valid it is sufficient to demand

$$
\frac{\omega \eta}{\Phi_{0}} \gg B_{0}(0) \lambda^{-2}, \quad H_{c 1} k_{z}^{2}, \quad H_{c 1} k^{2},
$$
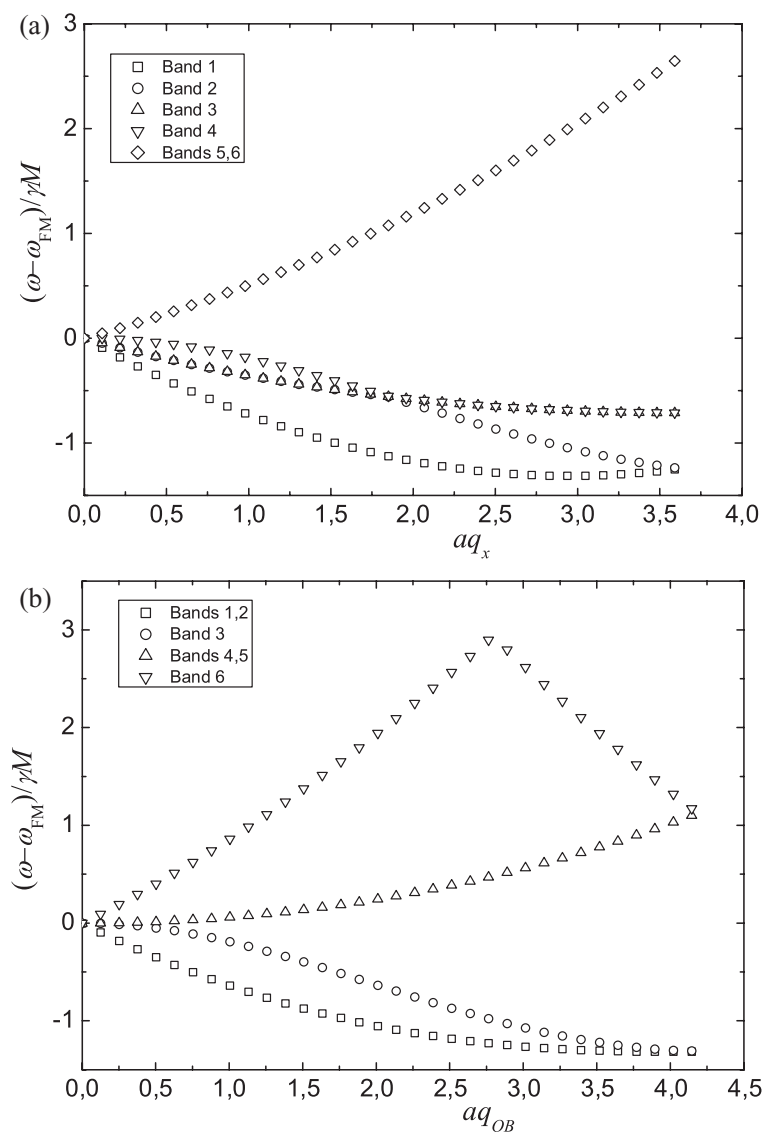

FIG. 8. The cross sections of the lowest six bands along the lines (a) $O C$ and (b) $O B \cdot B_{0}(0)=0.25 \beta$. where

$$
H_{c 1}=\frac{\Phi_{0}}{4 \pi \lambda^{2}} \ln \frac{\lambda}{\xi}
$$

is the first critical field. The conditions (49) can be satisfied in the clean limit: It is known that the viscosity increases with increasing normal state conductivity. ${ }^{19}$

Within our approximation

$$
\Delta \mathbf{R}=\sum_{G_{i}<\xi^{-1}} \frac{\Phi_{0} k_{z} \mathbf{m}\left(\mathbf{G}_{i}\right)}{\omega \eta\left[1+\lambda^{2}\left(k_{z}^{2}+q_{i}^{2}\right)\right]} .
$$

After substituting this into Eq. (28) we can repeat the calculations from Sec. II B and obtain the system (33) with

$$
\begin{aligned}
\omega_{0}\left(q, k_{z}\right)= & \gamma M\left(\tilde{K}+\alpha q^{2}-\frac{2 \pi\left(2 k_{z}^{2}+q^{2}\right)}{\lambda^{-2}+k_{z}^{2}+q^{2}}\right. \\
& \left.-\frac{i B_{0}(0) \Phi_{0} k_{z}^{2}}{\omega \eta\left(1+\lambda^{2}\left(k_{z}^{2}+q^{2}\right)\right)^{2}}\right), \\
b_{i j}= & \frac{B_{0}\left(\mathbf{G}_{i}-\mathbf{G}_{j}\right)}{M}-\frac{i B_{0}(0) \Phi_{0} k_{z}^{2}}{\omega \eta} \\
& \times \frac{1}{\left[1+\lambda^{2}\left(k_{z}^{2}+q_{i}^{2}\right)\right]\left[1+\lambda^{2}\left(k_{z}^{2}+q_{j}^{2}\right)\right]} .
\end{aligned}
$$

Equation (33) now represents an eigenvalue problem for a symmetric non-Hermitian matrix. Due to dissipation the magnetic excitation levels are broadened, which can lead to the smearing of the gaps between the energy bands. To observe the effects connected with the presence of the gap $\Delta_{i j}$ for $q_{i} \approx q_{j}$ we have to provide that

$$
\frac{B_{0}\left(\mathbf{G}_{i}-\mathbf{G}_{j}\right)}{M} \gtrsim \frac{B_{0}(0) \Phi_{0} k_{z}^{2}}{\omega \eta\left[1+\lambda^{2}\left(k_{z}^{2}+q_{i}^{2}\right)\right]^{2}} .
$$

This means that the viscosity should be sufficiently large, or the longitudinal wave number $k_{z}$ should be small so that vortex motion is not excited.

In metallic ferromagnets another important mechanism of dissipation exists, which is due to magnon-conduction electron scattering. ${ }^{20}$ This kind of dissipation can be qualitatively taken into account by introducing a phenomenological damping term in the right-hand side of the Landau-Lifshitz equation $(4)^{21}$

$$
\left(\frac{\partial \mathbf{M}}{\partial t}\right)_{\text {damp }}=-\gamma \nu \mathbf{M} \times \frac{\partial \mathbf{M}}{\partial t},
$$

where $v$ is a relaxation constant defining the magnetization relaxation time $\tau=(\gamma \nu M \omega)^{-1}$. The mentioned mechanism of dissipation does not smear out the gaps in the magnon spectrum if

$$
\tau^{-1} \lesssim \gamma B_{0}\left(\mathbf{G}_{i}-\mathbf{G}_{j}\right) .
$$

Data on the relaxation time $\tau$ in the U-based ferromagnetic superconductors are not available yet. The typical theoretical and experimental values for this quantity in ordinary metallic ferromagnets are $10^{-9}-10^{-8} s$ (see Chap. 5 in Ref. 20 and references therein). We can estimate the Fourier component of the vortex field as

$$
B_{0}\left(\mathbf{G}_{i}-\mathbf{G}_{j}\right) \sim \Phi_{0} / \lambda^{2} .
$$




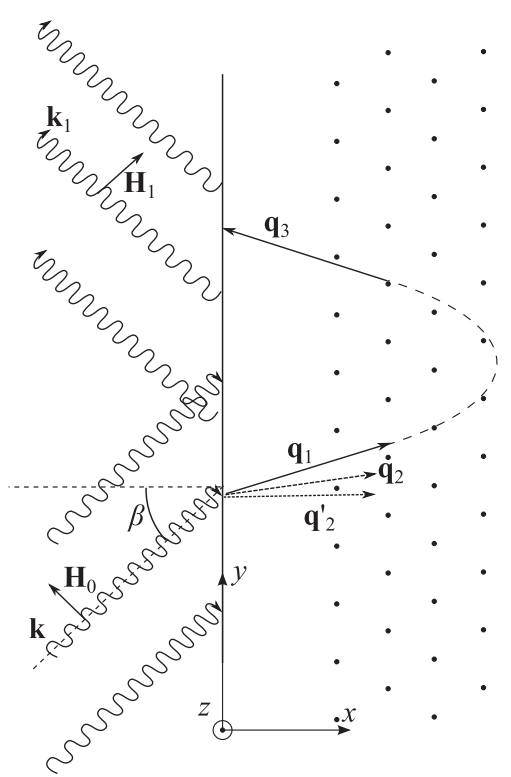

FIG. 9. An electromagnetic wave (k) incident on the flat surface of a ferromagnetic superconductor is partially reflected back as a wave with the wave vector $\mathbf{k}_{1}$. Inside the material one propagating $\left(\mathbf{q}_{1}\right)$ and two decaying magnon modes $\left(\mathbf{q}_{2}\right.$ and $\left.\mathbf{q}_{2}^{\prime}\right)$ are excited. The mode $\mathbf{q}_{1}$ undergoes Bragg reflection on the vortex lattice (represented by dots) and transforms into the mode $\mathbf{q}_{3}$ propagating towards the sample surface.

Hence, the frequency gap $\Delta=2 \gamma B_{0}\left(\mathbf{G}_{i}-\mathbf{G}_{j}\right)$ is of the order of $10^{-8} \mathrm{~s}^{-1}$. Thus, the relaxation rate $\tau^{-1}$ and the gap $\Delta$ appear to be of the same order of magnitude.

\section{MICROWAVE PROBING OF THE BAND STRUCTURE}

In this section we will demonstrate how the evidence of the gaps in the magnon spectrum can be found using microwave probing. Consider an electromagnetic Transverse Electric (TE) wave with the wave vector $\mathbf{k}$ and amplitude $\mathbf{H}_{0}$ incident on a ferromagnetic superconductor occupying the half-space $x>0$ (see Fig. 9). For simplicity, we assume $k_{z}=0$. Note that in a Transverse Magnetic (TM) wave the field $\mathbf{H}$ would oscillate along the direction of the uniform magnetization, hence, this wave does not excite magnons and is totally reflected. For this reason, we consider further a TE wave. Such a wave excites three magnon modes inside the ferromagnet: one propagating $\left(\mathbf{q}_{1}\right)$ and two decaying modes $\left(\mathbf{q}_{2}\right.$ and $\left.\mathbf{q}_{2}^{\prime}\right)$. The wave vectors of these modes are determined from the two equations

$$
\begin{gathered}
\omega^{2}=\omega_{0}^{2}(q)=\gamma^{2} M^{2}\left(K+\frac{B_{0}(0)}{M}+\alpha q^{2}\right) \\
\times\left(K+\frac{B_{0}(0)}{M}+\alpha q^{2}-\frac{4 \pi q^{2}}{q^{2}+\lambda^{-2}}\right), \\
q_{x}^{2}=q^{2}-k_{y}^{2},
\end{gathered}
$$

The propagating mode can be reflected back to the surface of the ferromagnet due to Bragg scattering on vortices, if two conditions are fulfilled for some wave vector $\mathbf{q}_{3}$

$$
\mathbf{q}_{3}=\mathbf{q}_{1}+\mathbf{G}, \quad \omega_{0}\left(q_{3}\right) \approx \omega_{0}\left(q_{1}\right),
$$

where $\mathbf{G}=-G \mathbf{x}_{0}$ is a vector of the reciprocal lattice (16).
We will determine the amplitude $\mathbf{H}_{1}$ of the reflected electromagnetic wave. For the evaluation of this amplitude the equilibrium field distributiton $\mathbf{B}_{\mathrm{eq}}(\mathbf{r})$ in the material is required

$$
\mathbf{B}_{\mathrm{eq}}=4 \pi M \mathbf{z}_{0} e^{-x / \lambda}+\mathbf{B}_{0}\left(\mathbf{r}-x_{v} \mathbf{x}_{0}\right)+\mathbf{B}_{0}^{\prime}(\mathbf{r}) .
$$

Here, the first term represents the screened intrinsic magnetic field (we assume that there is no constant external field $\mathbf{H}_{e}=$ $0), \mathbf{B}_{0}$ is the vortex field given by Eq. (15), $x_{v}$ specifies the shift of the vortex lattice with respect to the surface, and the term $\mathbf{B}_{0}^{\prime}(\mathbf{r})$ is responsible for the vortex lattice distortion in a surface layer with a thickness of the order of $\lambda$.

We consider a dense vortex lattice, so that $a \ll \lambda$. To observe the effects connected with Bragg reflection of magnons we have to demand $q_{1} \sim a^{-1}$, hence, $\alpha q_{1}^{2} \gg 1$. The nonstationary component of the magnetization can be presented in the form

$$
\mathbf{m} \approx \mathbf{m}_{1}(x) e^{i \mathbf{q}_{1} \mathbf{r}}+\mathbf{m}_{2} e^{i \mathbf{q}_{2} \mathbf{r}}+\mathbf{m}_{2}^{\prime} e^{i \mathbf{q}_{2}^{\prime} \mathbf{r}}+\mathbf{m}_{3}(x) e^{i \mathbf{q}_{3} \mathbf{r}},
$$

where $\mathbf{m}_{1}(x)$ and $\mathbf{m}_{3}(x)$ vary slowly in space. In the Appendix, using a simple perturbation theory, we demonstrate that the influence of the screened intrinsic field on the magnon modes is not essential. By similar reasons, the distortion field $\mathbf{B}_{0}^{\prime}(\mathbf{r})$ also does not affect significantly the spin wave amplitudes. Hence, we can consider $\mathbf{m}_{2}$ and $\mathbf{m}_{2}^{\prime}$ to be constant.

Now we write down the boundary conditions. Directly from Eq.(4) we obtain

$$
\frac{\partial \mathbf{m}}{\partial x}(x=0)=0
$$

The continuity condition for the tangential component of the magnetic field $\mathbf{H}$ reads

$\left(H_{0}+H_{1}\right) \cos \beta=-4 \pi \sum_{i} \frac{\left(\lambda^{-2}+k_{y}^{2}\right) m_{i y}(0)+k_{y} q_{i x} m_{i x}(0)}{q_{i}^{2}+\lambda^{-2}}$,

where summation is performed over all four modes. The electric field inside the material is

$$
\mathbf{e}=-4 \pi k \sum_{i} \frac{\mathbf{q}_{\mathbf{i}} \times \mathbf{m}_{i}}{q_{i}^{2}+\lambda^{-2}} e^{i \mathbf{q}_{i} \mathbf{r}} .
$$

The continuity condition for the electric field reads

$$
H_{1}-H_{0}=4 \pi k \sum_{i} \frac{k_{y} m_{i x}(0)-q_{i x} m_{i y}(0)}{q_{i}^{2}+\lambda^{-2}} .
$$

The wave number $q_{2}^{\prime}$ has a large modulus $\left(q_{2}^{\prime 2} \approx-2 \omega / \gamma M \alpha\right)$ as compared to the other wave numbers, and the corresponding magnetization component $\mathbf{m}_{2}^{\prime}$ is small. It can be neglected in Eqs. (59) and (61). To exclude $\mathbf{m}_{2}^{\prime}$ from Eq. (58), we note that $m_{2 x}^{\prime} \approx i m_{2 y}^{\prime}$, hence

$$
\sum_{i=1}^{3} q_{i x}\left(m_{i x}-i m_{i y}\right)=0
$$

or

$$
\sum_{i=1}^{3} q_{i x} m_{i y}=0
$$


since $m_{i x} \approx-i m_{i y}$ for $i=1,2,3$. Now we have to find a connection between $\mathbf{m}_{1}$ and the amplitude of the Braggreflected mode $\mathbf{m}_{3}$. In these modes the magnetic field is small as compared to $\alpha \nabla^{2} \mathbf{m}$, so the linearized Landau-Lifshitz equation can be simplified as follows:

$$
\frac{\omega}{\gamma M} \mathbf{m}=\tilde{K} \mathbf{m}-\alpha \nabla^{2} \mathbf{m}+\frac{1}{M} B_{0}\left(\mathbf{r}-x_{v} \mathbf{x}_{0}\right) \mathbf{m} .
$$

To find the link between the mentioned modes it is sufficient to conserve only two terms in the Fourier series of the vortex field

$$
B_{0}(G)\left(e^{i G\left(x-x_{v}\right)}+e^{-i G\left(x-x_{v}\right)}\right) .
$$

By substituting $\mathbf{m}=\mathbf{m}_{1}(x) e^{i \mathbf{q}_{1} \mathbf{r}}+\mathbf{m}_{3}(x) e^{i \mathbf{q}_{3} \mathbf{r}}$ into Eq. (63) and neglecting the second derivatives of $\mathbf{m}_{1}(x)$ and $\mathbf{m}_{3}(x)$, we obtain

$$
\begin{aligned}
& i v_{g x} \frac{\partial \mathbf{m}_{1}}{\partial x}=\frac{\Delta}{2} e^{-i \varphi-i \delta x} \mathbf{m}_{3}(x), \\
& i v_{g x} \frac{\partial \mathbf{m}_{3}}{\partial x}=-\frac{\Delta}{2} e^{i \varphi+i \delta x} \mathbf{m}_{1}(x),
\end{aligned}
$$

where

$$
v_{g x}=\frac{\partial \omega_{0}}{\partial q_{x}}\left(\mathbf{q}_{1}\right)=2 \gamma M \alpha q_{1 x},
$$

$\Delta=2 \gamma B(G)$ is the frequency gap [see Eq.(43)], $\varphi=G x_{v}$, and $\delta=2 q_{1 x}-G$. The two linearly independent solutions of Eqs. (64) and (65) are

$$
\begin{aligned}
\mathbf{m}_{1}(x) & =\left(\mathbf{x}_{0}+i \mathbf{y}_{0}\right) e^{(\epsilon-i \delta / 2) x}, \\
\mathbf{m}_{3}(x) & =\frac{2 i v_{g x} e^{i \varphi}}{\Delta}\left(\epsilon-i \frac{\delta}{2}\right)\left(\mathbf{x}_{0}+i \mathbf{y}_{0}\right) e^{(\epsilon+i \delta / 2) x}, \\
\epsilon & = \pm \frac{1}{2} \sqrt{\frac{\Delta^{2}}{v_{g x}^{2}}-\delta^{2}} .
\end{aligned}
$$

For $|\delta|<\Delta / v_{g x}$ we reject the growing solution, selecting the minus sign in Eq. (66). For $|\delta|>\Delta / v_{g x}$ we select the solution where $\left|\mathbf{m}_{1}\right| \gg\left|\mathbf{m}_{3}\right|$ when $|\delta| \gg \Delta / v_{g x}$

$$
\begin{aligned}
& \epsilon=\frac{i}{2} \sqrt{\delta^{2}-\frac{\Delta^{2}}{v_{g x}^{2}}} \quad \text { for } \quad \delta>0, \\
& \epsilon=-\frac{i}{2} \sqrt{\delta^{2}-\frac{\Delta^{2}}{v_{g x}^{2}}} \quad \text { for } \quad \delta<0 .
\end{aligned}
$$

This choice of the sign allows to reject the solution with the negative $x$ component of the group velocity. At $x=0$ we have

$$
\mathbf{m}_{3}(0)=A \mathbf{m}_{1}(0)
$$

$$
A=\frac{2 i v_{g x} e^{i \varphi}}{\Delta}\left(\epsilon-i \frac{\delta}{2}\right) .
$$

Now we are ready to write the system of linear equations which will allow us to determine the amplitude of the reflected wave $H_{1}$. Equations (59), (61), (62), and (68) yield

$$
\tilde{q}_{1 x} \tilde{m}_{1 y}+q_{2 x} m_{2 y}=0
$$

$$
\begin{gathered}
4 \pi \frac{i k_{y} \tilde{q}_{1 x}-\lambda^{-2}-k_{y}^{2}}{q_{1}^{2}+\lambda^{-2}} \tilde{m}_{1 y}+4 \pi \frac{i k_{y} q_{2 x}-\lambda^{-2}-k_{y}^{2}}{q_{2}^{2}+\lambda^{-2}} m_{2 y} \\
=\left(H_{0}+H_{1}\right) \cos \beta \\
-4 \pi k \frac{i k_{y}+\tilde{q}_{1 x}}{q_{1}^{2}+\lambda^{-2}} \tilde{m}_{1 y}-4 \pi k \frac{i k_{y}+q_{2 x}}{q_{2}^{2}+\lambda^{-2}} m_{2 y}=H_{1}-H_{0}
\end{gathered}
$$

where

$$
\tilde{m}_{1 y}=(A+1) m_{1 y}, \quad \tilde{q}_{1 x}=\frac{1-A}{1+A} q_{1 x} .
$$

Note that the problem of electromagnetic wave reflection from a superconductor in the mixed state is formally equivalent to the same problem for a superconductor without vortices with the only difference that $q_{1 x}$ is replaced by $\tilde{q}_{1 x}$. From the systems (70) to (72) we find the reflection coefficient

$$
R=\frac{H_{1}}{H_{0}}=\frac{\frac{\lambda^{-2} q_{2 x}}{q_{1}^{2}+\lambda^{-2}}-\frac{\alpha\left(q_{1}^{2}+\lambda^{-2}\right)}{2 \pi} \tilde{q}_{1 x}+e^{-i \beta}\left(k q_{2 x} \frac{i k_{y}+\tilde{q}_{1 x}}{q_{1}^{2}+\lambda^{-2}}-k \tilde{q}_{1 x} \frac{\left(i k_{y}+q_{2 x}\right) \alpha\left(q_{1}^{2}+\lambda^{-2}\right)}{2 \pi \lambda^{-2}}\right)}{\frac{\lambda^{-2} q_{2 x}}{q_{1}^{2}+\lambda^{-2}}-\frac{\alpha\left(q_{1}^{2}+\lambda^{-2}\right)}{2 \pi} \tilde{q}_{1 x}-e^{i \beta}\left(k q_{2 x} \frac{i k_{y}+\tilde{q}_{1 x}}{q_{1}^{2}+\lambda^{-2}}-k \tilde{q}_{1 x} \frac{\left(i k_{y}+q_{2 x}\right) \alpha\left(q_{1}^{2}+\lambda^{-2}\right)}{2 \pi \lambda^{-2}}\right)},
$$

where we used the relation

$$
\alpha q_{1}^{2}-\frac{2 \pi q_{1}^{2}}{q_{1}^{2}+\lambda^{-2}}=\alpha q_{2}^{2}-\frac{2 \pi q_{2}^{2}}{q_{2}^{2}+\lambda^{-2}},
$$

which is valid in the large anisotropy limit. The expression for the reflectivity coefficient can be simplified, if we take into account that $q_{1} \gg \lambda^{-1}$ and $q_{2 x} \approx i \lambda^{-1}$

$$
R=\frac{1+i k^{2} \lambda^{2} e^{-i \beta} \sin \beta+Q\left(i-k \lambda e^{-i \beta}\right)}{1-i k^{2} \lambda^{2} e^{i \beta} \sin \beta+Q\left(i+k \lambda e^{i \beta}\right)},
$$

where

$$
Q=\frac{\alpha \lambda^{2} q_{1}^{4}}{2 \pi} q_{1 x} \lambda \frac{1-A}{1+A} .
$$

In Eq. (75) we dropped small terms which have a negligible effect on the modulus of the reflectivity coefficient. Since the quantity $A$ can take any value within the circle $|A| \leqslant 1$, so the only restriction on $Q$ is $\operatorname{Re} Q \geqslant 0$.

When $|A|=1, Q$ is purely imaginary, and $|R|=1$, i.e., the wave is totally reflected. This is explained by the fact that in this range of parameters the frequency $\omega$ is within the frequency gap, and magnons cannot propagate in the sample.

Consider now frequencies far from the gap: $\delta \gg \Delta / v_{g_{x}}$. In this case the magnons do not interact with the vortex lattice, and the quantity $Q$ is real and large: $Q \gg 1$. From Eq. (75) we obtain

$$
1-|R|^{2}=\frac{4 k \lambda \cos \beta}{Q} \ll 1
$$


An interesting effect which follows from Eq. (75) is the complete transmission of the wave for a frequency close to the frequency gap. Let us put $R=0$. Then

$$
Q \approx i+k \lambda e^{-i \beta} \text {. }
$$

This is possible when $A \approx 1$ and $|A|<1$, i.e., the detuning from the gap must be very small. For example, if $k \alpha \lambda^{4} q_{1}^{5} \gg 1$

$$
\frac{|\delta| v_{g x}}{\Delta}-1 \approx \frac{8 \pi^{2} k^{2} \cos ^{2} \beta}{\alpha^{2} \lambda^{4} q_{1}^{10}} .
$$

Note that for $A \approx 1 \mathbf{m}_{1}(0) \approx \mathbf{m}_{3}(0)$. The effect of complete transmission is related to a similar effect in a Fabri-Perrot resonator: In our system, the surface of the material and the vortex lattice play the roles of the first and second mirrors, respectively.

We need to stress that the system must be finely tuned to make the dip in the $R(\omega)$ dependence observable. Indeed, the parameter $A$ must be equal to unity on the border of the gap, which imposes a constraint on the parameters $G$ and $x_{v}$ :

$$
e^{i G x_{v}}= \pm 1 \text {. }
$$

This condition may be satisfied by applying an external magnetic field.

\section{CONCLUSION}

By solving the London and Landau-Lifshitz equations we investigated the magnon spectrum of a ferromagnetic superconductor in the mixed state. The case of a large easy-axis magnetocrystalline anisotropy has been considered, which is relevant to the U-based compounds. ${ }^{1,2,4}$ We proved that the magnon spectrum has a Bloch-like band structure due to the presence of the periodic vortex field. For sufficiently small intervortex distances $a \lesssim \lambda$, the gaps between adjacent bands can be calculated using an analog of the weak-binding approximation. These gaps are proportional to the Fourier components $B_{0}(\mathbf{G})$ of the unperturbed vortex field [see Eq. (43)]. If the material has isotropic properties in the plane perpendicular to the easy $z$ axis, some bands may intersect in points of high symmetry of the Brillouin zone. For spin waves having a nonzero $z$ component of the wave vector the band structure is smeared out in dirty materials because of dissipation connected with viscous vortex motion.

Using numerical calculations, we demonstrated that the band structure changes qualitatively with varying applied field. For example, when the average field becomes smaller than $\beta / 4$ [see Eq. (48)] the smooth maximum in the center of the lowest band transforms into a rather sharp peak (see Figs. 3 to 7). This effect appears due to the nonmonotonicity of the spectrum in the Meissner state.
We propose to probe the energy gaps by measuring the frequency dependence of the reflectivity coefficient $R(\omega)$ for an electromagnetic wave incident on the flat surface of a ferromagnetic superconductor. For frequencies lying inside the gap a maximum of $|R(\omega)|$ should be observable. Also, for a very small detuning from the gap, the reflectivity coefficient may exhibit a narrow dip. The knowledge of the gap frequencies allows to determine with high accuracy such parameters as the anisotropy coefficient $K$ and the exchange constant $\alpha$.

Finally, note that the self-induced vortex state in ferromagnetic superconductors may provide a negative permeability at frequencies near the ferromagnetic resonance, thus making the ferromagnetic superconductors potential candidates for metamaterials design.

\section{ACKNOWLEDGMENTS}

We are thankful to A. S. Mel'nikov and L. N. Bulaevskii for helpful discussion. This work was supported, in part, by European IRSES program SIMTECH (Contract No. 246937), the Russian Foundation for Basic Research, FTP "Scientific and educational personnel of innovative Russia in 2009-2013", the French ANR program MASH, and LabEx "Amadeus" program.

\section{APPENDIX}

In this Appendix we consider the modification of the mode with the wave vector $\mathbf{q}_{2}$ in the vicinity of the surface. This consideration can be simply generalized for all other modes.

First, we note that $q_{2}^{2} \approx-\lambda^{-2}$ and $q_{2 x} \approx i \lambda^{-1}$ since $\left|k_{y}\right| \ll$ $\lambda^{-1}$ (this inequality holds for frequencies, which are much smaller than the plasma frequency). We substitute into Eq. (17) $B_{0}=4 \pi M e^{-x / \lambda}$, and $\mathbf{m} \approx \mathbf{m}_{2} e^{-x / \lambda}+\mathbf{m}_{2}^{(1)} e^{-2 x / \lambda}$, where $\mathbf{m}_{2}^{(1)}$ is the amplitude of the first-order correction, which is to be estimated. This correction is determined from the following equations:

$$
\begin{aligned}
-\frac{i \omega}{\gamma M} \mathbf{m}_{2}^{(1)}+\left(K-4 \alpha \lambda^{-2}\right) \mathbf{m}_{2}^{(1)} \times \mathbf{z}_{0} & =\left(\mathbf{b}_{2}^{(1)}-4 \pi \mathbf{m}_{2}\right) \times \mathbf{z}_{0}, \\
-\frac{3}{\lambda^{2}} \mathbf{b}_{2}^{(1)} & =-\frac{16 \pi}{\lambda^{2}} m_{2 y}^{(1)} \mathbf{y}_{0} . \quad(\mathrm{A} 1)
\end{aligned}
$$

For $\omega \approx \gamma M\left(K+\alpha q_{1}^{2}\right), \alpha q_{1}^{2} \gg 1$ we find

$$
\mathbf{m}_{2}^{(1)} \approx \frac{4 \pi}{\alpha q_{1}^{2}} \mathbf{m}_{2} .
$$

Hence, $\left|\mathbf{m}_{2}^{(1)}\right| \ll\left|\mathbf{m}_{2}\right|$, so the small correction can be neglected.
${ }^{1}$ S. S. Saxena, P. Agarwal, K. Ahilan, F. M. Grosche, R. K. W. Haselwimmer, M. J. Steiner, E. Pugh, I. R. Walker, S. R. Julian, P. Monthoux, G. G. Lonzarich, A. Huxley, I. Sheikin, D. Braithwaite, and J. Flouquet, Nature (London) 406, 587 (2000). ${ }^{2}$ Dai Aoki, Andrew Huxley, Eric Ressouche, Daniel Braithwaite, Jacques Flouquet, Jean-Pascal Brison, Elsa Lhotel, and Carley Paulsen, Nature (London) 413, 613 (2001).
${ }^{3}$ T. C. Kobayashi, S. Fukushima, H. Hidaka, H. Kotegawa, T. Akazawa, E. Yamamoto, Y. Haga, R. Settai, and Y. Onuki, Physica B 378-380, 355 (2006).

${ }^{4}$ N. T. Huy, A. Gasparini, D. E. de Nijs, Y. Huang, J. C. P. Klaasse, T. Gortenmulder, A. de Visser, A. Hamann, T. Görlach, and H. v. Löhneysen, Phys. Rev. Lett. 99, 067006 (2007).

${ }^{5}$ J. Floquet and A. Buzdin, Phys. World 15, 41 (2002). 
${ }^{6}$ V. Braude and E. B. Sonin, Phys. Rev. Lett. 93, 117001 (2004).

${ }^{7}$ A. I. Buzdin, JETP Lett. 40, 956 (1984) [Pis'ma v ZhETF 40, 193 (1984)].

${ }^{8}$ V. Braude and E. B. Sonin, Europhys. Lett. 72, 124 (2005).

${ }^{9}$ V. Braude, Phys. Rev. B 74, 054515 (2006).

${ }^{10}$ N. A. Logoboy and E. B. Sonin, Phys. Rev. B 75, 153206 (2007).

${ }^{11}$ L. N. Bulaevskii, M. Hruška, and M. P. Maley, Phys. Rev. Lett. 95, 207002 (2005).

${ }^{12}$ A. Shekhter, L. N. Bulaevskii, and C. D. Batista, Phys. Rev. Lett. 106, 037001 (2011).

${ }^{13}$ Shi-Zeng Lin and Lev N. Bulaevskii, Phys. Rev. B 85, 134508 (2012).

${ }^{14}$ S.-Z. Lin, L. N. Bulaevskii, and C. D. Batista, Phys. Rev. B 86, 180506(R) (2012).
${ }^{15}$ T. K. Ng and C. M. Varma, Phys. Rev. B 58, 11624 (1998).

${ }^{16}$ Vu Hung Dao, Sebastien Burdin, and Alexandre Buzdin, Phys. Rev. B 84, 134503 (2011).

${ }^{17}$ A. B. Shick, Phys. Rev. B 65, 180509 (2002).

${ }^{18}$ N. T. Huy, D. E. de Nijs, Y. K. Huang, and A. de Visser, Phys. Rev. Lett. 100, 077002 (2008).

${ }^{19}$ V. V. Schmidt, The Physics of Superconductors: Introduction to Fundamentals and Applications (Springer-Verlag, Berlin, 1997).

${ }^{20} \mathrm{~S}$. V. Vonsovskii, Ferromagnetic resonance: The phenomenon of resonance absorption of $\mathrm{HF}$ electromagnetic field in ferromagnetic materials (Israel Program for Scientific Translations, Jerusalem, 1964).

${ }^{21}$ T. L. Gilbert, IEEE Trans. Magn. 40, 3443 (2004). 